Supporting Information

\title{
Synthesis of Nanosheets Containing Uniformly Dispersed PdI ions at an Aqueous/Aqueous Interface: Development of a Highly Active Nanosheet Catalyst for Mizoroki-Heck Reaction
}

Daisuke Nagai, ${ }^{\star}+\mp$ Moeko Morita, ${ }^{\dagger}$ and Takeshi Yamanobe ${ }^{\dagger}$

\footnotetext{
† Division of Molecular Science, Faculty of Science and Technology, Gunma University, 1-5-1 Tenjin-cho, Kiryu, Gunma Prefecture 376-8515, Japan

$\$$ Present address: School of Food and Nutritional Science, University of Shizuoka, 52-1 Yada, Shizuoka-shi 422-8526, Japan
} 
Correspondence to: D. Nagai (E-mail: daisukenagai@u-shizuoka-ken.ac.jp, Tel \& Fax: +81$52-264-5729)$

\section{Experimental procedure for the control experiment of Mizoroki-Heck reaction}

NMP (5.00 mL), iodobenzene $(0.540 \mathrm{~mL} .5 .00 \mathrm{mmol})$, methyl acrylate $(0.540 \mathrm{~mL}, 6.00$ $\mathrm{mmol}), \mathrm{Pd}^{\mathrm{II}}$ nanosheet $\left(11.4 \mathrm{mg}, 1.5 \times 10^{-3} \mathrm{mmol}\right.$ of $\mathrm{Pd}, 0.03 \mathrm{~mol} \%$ of Pd to idobenzene), and undecane $(0.4 \mathrm{~mL}$ as an internal standard) were added to a glass ampule under nitrogen. The glass ampule was sealed and stirred at $140^{\circ} \mathrm{C}$ for 15 min to reach $54 \%$ yield of the product, which was confirmed by gas chromatography $(\mathrm{GC})$ analysis. The glass ampule was broken, and $\mathrm{Pd}^{\mathrm{II}}$ nanosheet was removed by membrane filtration (pore size $=0.45 \mu \mathrm{m}$ ), and the filtrate was added into a glass ample under nitrogen again. The glass ampule was sealed and stirred at $140^{\circ} \mathrm{C}$ for an additional $20 \mathrm{~h}$. GC analysis of the filtrate confirmed that the reaction did not proceed further. An aliquot $(0.250 \mathrm{~mL})$ of the filtrate was removed for sampling. After appropriate dilution, the $\mathrm{Pd}^{\mathrm{II}}$ concentration in the filtrate was determined by AAS.

Scanning probe microscopy (SPM) of the nanosheets on a silicon substrate showed a thickness of approximately $117 \mathrm{~nm}$ (Figure S1).
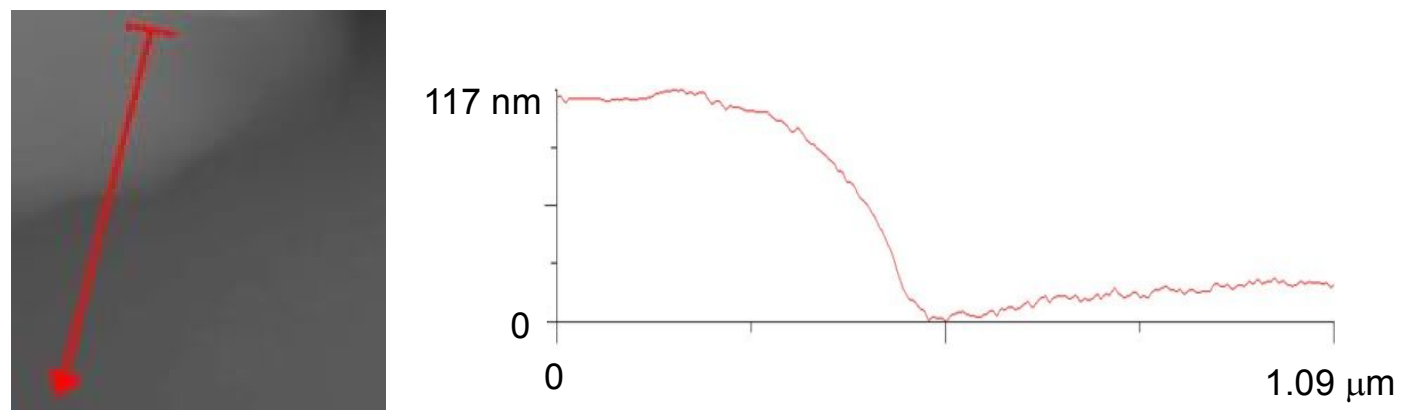

Figure S1. Scanning electron microscopy image of a nanosheet on a Si substrate. 
The inverse addition of a dispersed aqueous solution of HPMC to an aqueous solution of PdII ions did not generate the interface and the gels sunk into the underlying aqueous $\mathrm{Pd}^{\mathrm{II}}$ layer (Figure S2).
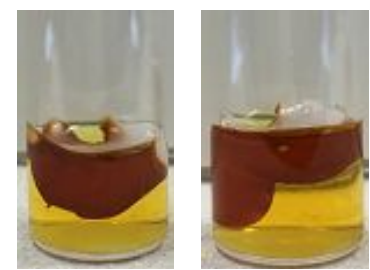

$12 \mathrm{mM}$
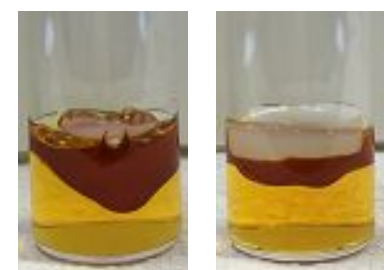

$16 \mathrm{mM}$

$18 \mathrm{mM}$
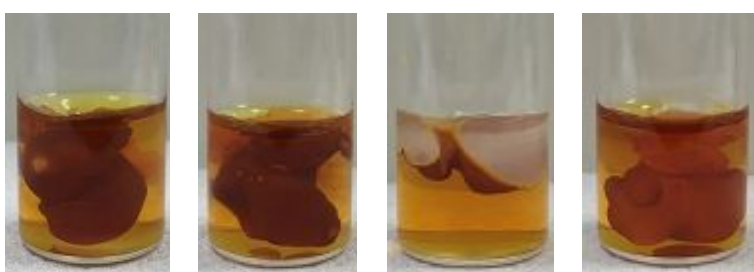

$22 \mathrm{mM}$

Figure S2. Photographs of bottom-up synthesis of nanosheets by addition of dispersed aqueous solutions of HPMC (30 wt $\%)$ to different concentrations of aqueous solutions of $\mathrm{Pd}^{\mathrm{II}}$ ion (12 34 $\mathrm{mM})$.

Electron probe micro analyzer (EPMA) mapping image of the nanosheet showed that the Pd species (blue dots) were uniformly dispersed in the nanosheets (Figure S3).

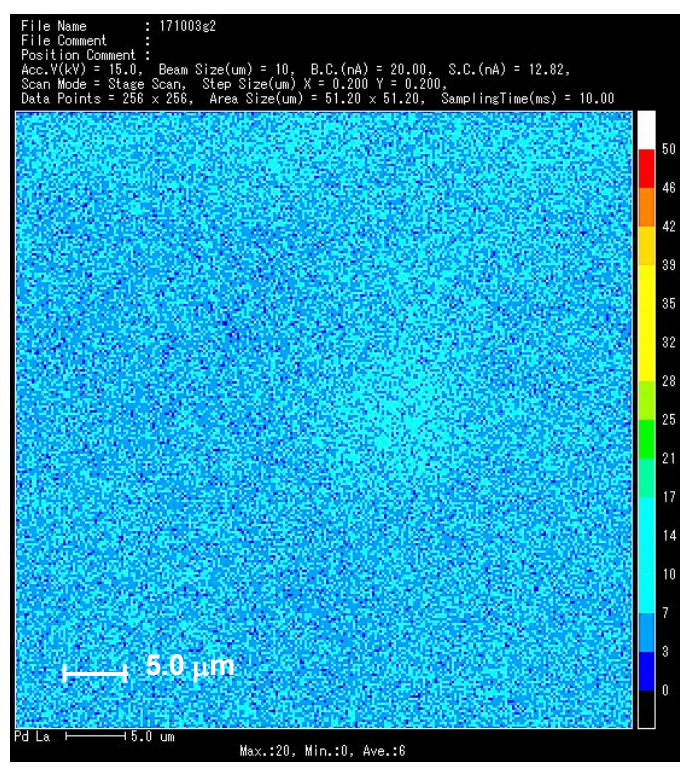

Figure S3. Electron probe micro analyzer (EPMA) mapping image of Pd $\mathrm{d}^{\mathrm{II}}$ nanosheet. 
As shown in Figure S4, the nanosheet morphology was maintained after the rection, which was confirmed by TEM analysis.

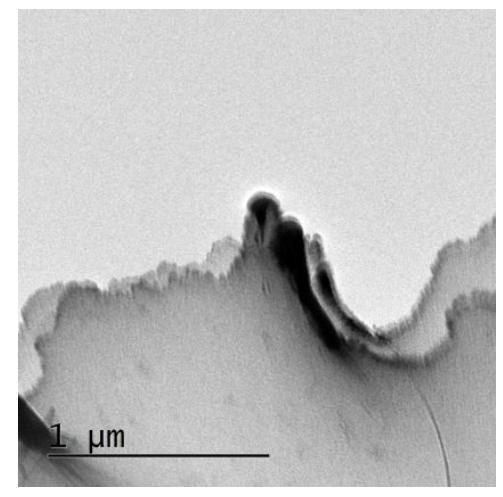

$1 \mu \mathrm{m}$

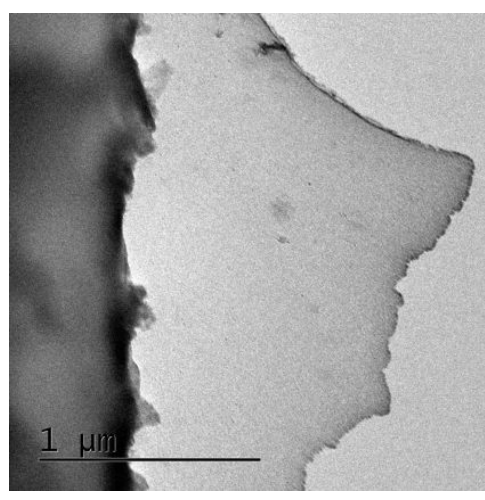

$1 \mu \mathrm{m}$

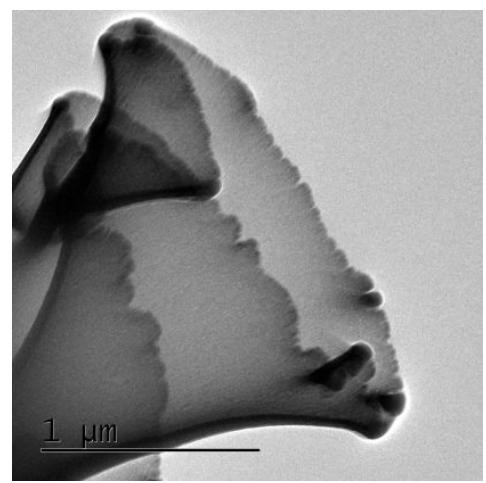

$1 \mu \mathrm{m}$

Figure S4. TEM images of $\mathrm{Pd}^{\mathrm{II}}$ nanosheets after the Mizoroki-Heck cross-coupling reaction.

As shown in Figure S5, the structure of the nanosheet was not disrupted, which was confirmed by TEM analysis.

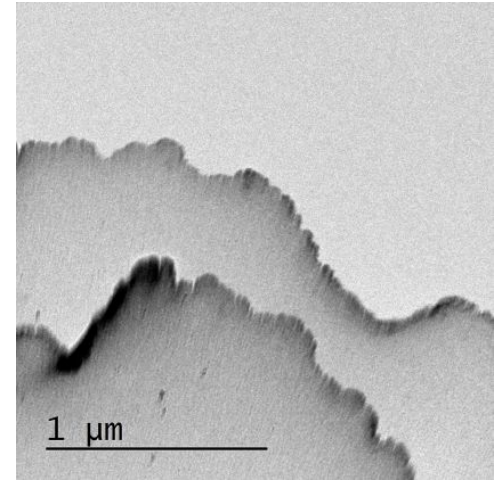

$1 \mu \mathrm{m}$

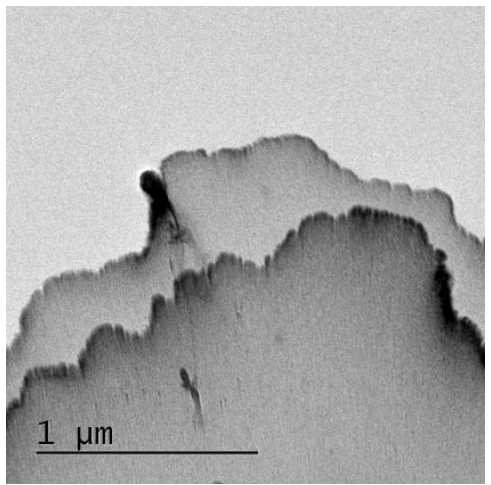

$1 \mu \mathrm{m}$

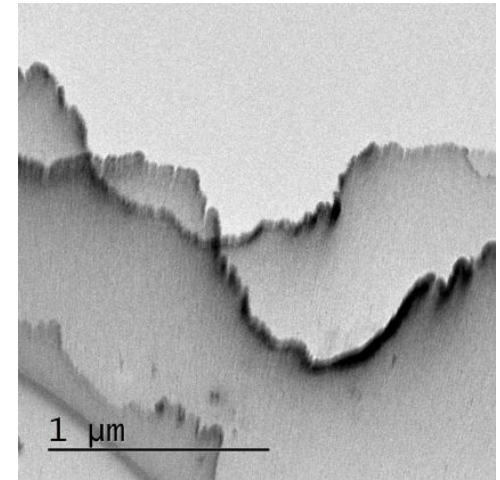

$1 \mu \mathrm{m}$

Figure S5. TEM images of $\mathrm{Pd}^{\mathrm{II}}$ nanosheets after cross-linking by hexamethylene diisocyanate. 